\title{
Advantage of a baculovirus expression system for protein-protein inter- action studies. Involvement of posttranslational phosphorylation in the interaction between wt p53 protein and poly(ADP-ribose) polymerase-1^
}

\author{
Gerald Schmid ${ }^{\star}$, Jacek Wojciechowski and Józefa Węsierska-Gądek ${ }^{\otimes}$
Cell Cycle Regulation Group, Division: Institute of Cancer Research, Department of Medicine I, Vienna Medical University, Vienna, Austria; ${ }^{\circledR}$-mail: Jozefa.Gadek-Wesierski@meduniwien.ac.at

Received: 15 March, 2005; revised: 23 May, 2005; accepted: 28 May, 2005

available on-line: 04 August, 2005

\begin{abstract}
We recently observed an interaction between poly(ADP-ribose) polymerase-1 (PARP-1) and the tumor suppressor p53 protein. However, more extensive studies on both proteins, especially those on characterization of their domains involved in the interaction were difficult due to very low expression levels of p53 in mammalian cells. Therefore, we generated recombinant proteins for such studies. To clarify which domains of human PARP-1 and of human wild-type (wt) p53 were involved in this protein-protein interaction, we generated baculoviral constructs encoding full length or distinct functional domains of both proteins. Full length PARP-1 was simultaneously coexpressed in insect cells with full length wt p53 protein or its distinct truncated fragments and vice versa. Reciprocal immunoprecipitation of Sf9 cell lysates revealed that the central and carboxy-terminal fragments of p53 each were sufficient to confer binding to PARP-1, whereas the amino-terminal part harbouring the transactivation functional domain was dispensable. On the other hand, the amino-terminal and central fragments of PARP-1 were both necessary for complex formation with $\mathrm{p} 53$ protein. Since the most important features of p53 protein are regulated by phosphorylation, we addressed the question whether its phosphorylation is essential for the binding between the two proteins. Baculovirally expressed wt p53 was post-translationally modified. At least six distinct p53 isomers were resolved by immunoblotting following two-dimensional separation of baculovirally expressed wt p53 protein. Using specific phospho-serine antibodies, we identified phosphorylation of baculovirally expressed p53 protein at five distinct sites. To define the role of p53 phosphorylation, pull-down assays using untreated and dephosphorylated p53 protein were performed. Dephosphorylated p53 failed to bind PARP-1, indicating that complex formation between the two proteins was regulated by phosphorylation of p53. The marked phosphorylation of p53 at Ser392 observed in unstressed cells suggests that the phosphorylated carboxy-terminal part of p53 undergoes complex formation with PARP-1 resulting in masking of the NES and thereby preventing its export.
\end{abstract}

Keywords: p53 phosphorylation, p53 stability, p53 nuclear export, NES, nucleocytoplasmic shuttling, cell cycle arrest, FACS analysis, 2D-PAGE, p53 isomers, p53 pull-down assay

The tumor suppressor protein p53 is one of the key players in cell cycle regulation, DNA damage response and apoptosis (for reviews, see Mowat 1998; Bargonetti \& Manfredi, 2002). Additionally, an important role of p53 and its homologues has been described in organismal development and recently, p53 has also been brought into context with cellular and organismal ageing (Donehower, 2002; Maier et al., 2004). Due to this wide range of fundamental functions a very tight spatial and temporal regulation of the protein is of paramount importance. The expression level and activity of p53 protein is regulated according to the developmental and physiological status of the particular cell, the protein being maintained at a very low level and occurring predominantly in its inactive form in unstressed cells.

^Presented at the International Review Conference on Biotechnology, Vienna, Austria, November 2004.

Present address:LMU Klinikum Grosshadern; Experimentelle Forschung Chirurgie, Muenchen, Germany.

Abbreviations: ECL, enhanced chemiluminescence; FCS, fetal calf serum; HRP, horseradish peroxidase; IP, immunoprecipitation; lacZ; $\beta$-galactosidase gene; MDM2, mouse double minute2; NES, nuclear export signal; NLS, nuclear localization signal; PARP-1, poly(ADP-ribose) polymerase-1; PBS, phosphate-buffered saline; WB, Western blot; wt, wild-type; X-Gal, 5-bromo-4-chloro-3-indolyl- $\beta$-D-galactopyranoside 
The tight regulation of wt p53 protein is achieved via four independent cellular mechanisms: the expression level of the protein, its stability, regulation of activity, and also by its spatial distribution. The expression of the protein is regulated by specific cofactors of the transcriptional machinery as a result of various extra- and intracellular stimuli. The required stability of the protein is achieved by an interplay of degrading and stabilizing mechanisms which are influenced through covalent modifications of the protein (for a review, see Appella \& Anderson, 2001). The key regulator for p53 stability is the Mdm2 protein which has an E3 ubiquitin ligase activity and is able to target p53 for ubiquitin-dependent proteolytic degradation (Momand et al., 1992; Haupt et al., 1997; Kubbutat et al., 1997). Additionally, a variety of proteins has been shown to be involved in the fine tuned regulation of the tumor suppressor. Among those poly(ADP-ribose) polymerase-1 (WesierskaGadek et al., 1999, 2000) and JNK kinase (Fuchs et al., 1998) are worth mentioning. p53 protein is able to switch between an active and a latent form. In its active form the protein constitutes a homo-tetramer and is able to carry out its function as a transcription factor. The intracellular distribution of p53 changes according to the cell cycle stage (Shaulsky et al., 1990). In normal unstressed cells p53 is primarily localized to the nucleus during the $G_{1}$ and the $\mathrm{G}_{2}$ phase and is predominantly cytoplasmic during $\mathrm{G}_{2}$ and $\mathrm{S}$ phases (Shaulsky et al., 1990). The intracellular distribution of p53 is consistent with its function as a checkpoint regulator of the cell cycle.

As a consequence to unfavourable intra- and extra-cellular conditions various stimuli induce the expression, stabilization and activation of p53. Obviously, several types of posttranslational modifications are involved in the regulation of p53 stability. Ubiquitination induced by $\mathrm{Mdm} 2$ protein leads to proteolytic degradation of p53 whereas phosphorylation of specific serine residues renders the protein resistant to this form of degradation. Depending on the negative stimuli and their severity p53 protein is able to induce downstream targets which lead to cell cycle arrest or, when the damage is too detrimental, to apoptosis. In the case of cell cycle arrest the cell is granted the opportunity to repair the damage and recover. In case the damage turns out to be too severe, the cell will enter a terminal cell cycle arrest.

PARP-1, a protein which directly binds to p53 (Wesierska-Gadek et al., 1996a) and regulates its stability (Wesierska-Gadek et al., 1999, Wesierska-Gadek \& Schmid, 2000) is also involved in DNA damage response and apoptosis (Berger 1985; de Murcia \& Menissier-de Murcia, 1994; D’Amours et al., 1999). To gain better insight into the interaction between the two proteins we mapped the domains of p53 and PARP-1 that are involved in the dimerization. The expression of high levels of wt p53 in mammalian cells would lead to cell cycle arrest and apoptosis, therefore, we chose the baculovirus expression system in insect cells. We expressed full length PARP-1 or p53 and various domains of either protein in those insect cells and used them to map the regions involved in heterodimerization (Wesierska-Gadek et al., 2003a). The phosphorylation pattern of p53 in insect cells is presumably identical to that in mammalian cells. This allowed us to also investigate the significance of p53 phosphorylation for its ability to bind PARP-1 (Wesierska-Gadek et al., 2003b). Using specific phosphoserine antibodies we were able to detect different p53 isoforms. Dephosphorylated p53 was unable to bind PARP-1 whereas the phosphorylation of Ser392 of p53 rendered the protein capable of binding PARP-1. This complex formation led to the masking of the NES of p53 and, thereby, prevented its nuclear export. Our data indicate that PARP-1 is involved in the stabilisation of the tumor suppressor protein p53.

\section{MATERIAL AND METHODS}

Cell lines. Spodoptera frugiperda (Sf9) insect cells were used for expression of baculovirally encoded human p53 and human PARP-1 constructs.

Antibodies. Different anti-p53 antibodies recognizing distinct epitopes were used. Monoclonal anti-p53 antibodies recognizing an epitope in the amino-terminus (DO-1 and DO-7), in the core domain (PAb240) or at the carboxy-terminus (PAb421) were from Oncogene Research Products (Cambridge, MA, USA). The polyclonal anti-p53 antibody CM-1 was obtained from Novocastra (Newcastle-upon-Tyne, UK). Additionally, monoclonal antibodies to phosphoserine (clone 4A9) were from Alexis Biochemicals Corp. (San Diego, CA, USA). Polyclonal antibodies against phospho-Ser15-, phospho-Ser20-, phosphoSer37-, phospho-Ser46-, and phospho-Ser392-p53 were from New England Biolabs (Beverly, MA, USA).

Polyclonal antibodies (N-20) specific for the amino-terminus of PARP-1 (aa 1-20) were from Santa Cruz (Santa Cruz, CA, USA). Monoclonal antiPARP-1 antibodies (C-2-10) and F-1-2-3 were from Dr. G. Poirier (STE-FOY, Canada) and F-2 was from Santa Cruz. Additionally, rabbit polyclonal antihuman PARP-1 antibodies from Boehringer (now Roche, Vienna, Austria) were used.

Monoclonal X-press antibodies directed against a 15 amino-acid motif present in recombinant proteins or against the His-tag sequence were obtained from Invitrogen Life Technologies (San Diego, CA, USA) and from Roche (Vienna, Austria), respectively. Immune complexes were detected using appropriate secondary antibodies linked to horseradish peroxidase (HRP) from Amersham International (Little Chalfont, Buckinghamshire, England) or from Pierce (Rockford, IL, USA). 
Cloning of p53 and PARP-1. All cDNAs were cloned into a modified baculovirus recombination vector (pBlueBacHis2B; Invitrogen Life Technologies, San Diego, CA, USA). One nucleotide in the vector was changed as follows: at position 166 a $\mathrm{G}$ was replaced by an A generating a BglII restriction site at position 167 without changing the amino-acid sequence of the His-tag. The resulting vector was called pBlueBacHis2BGS and all mentioned cDNAs were cloned into this newly generated $B g l I I$ site at their $5^{\prime}$ ends and into the SalI or HindIII restriction site at their $3^{\prime}$ ends. To generate the appropriate restriction sites flanking the coding region, PCR reactions were performed using appropriate primers as previously described (Wesierska-Gadek et al., 2003a). The annealing temperatures of the PCR reactions were $48-56^{\circ} \mathrm{C}$. Resulting cDNAs were digested and cloned into pBlueBacHis2BGS. The DNAs were sequenced in order to verify the correct cloning.

Transfection of insect cells. For transfection $4 \mu \mathrm{g}$ of the pBlueBacHis2BGS vector containing a p53 or PARP-1 insert were mixed with $1 \mu \mathrm{g}$ of the Bac-N-Blue plasmid, $1 \mathrm{ml}$ insect cell medium (without FCS) and $20 \mu \mathrm{l}$ insectin liposomes (all from Invitrogen Life Technologies, San Diego, CA). After recombination of the two plasmids, polyhedrin, a gene necessary for the creation of viral particles was reconstituted as well as a functional lac Z gene. The insectin liposomes facilitated successful transfection of the Sf9 cells incubated with the transfection mixture. After approx. 3 days the baculovirus started to lyse the insect cells and one week after transfection the supernatant including the newly formed virus particles was saved for subsequent infection of Sf9 cells. For selection, serial dilutions of the virus suspension (e.g. 1000 to 100000 fold) were put into petri dishes (10 cm diameter) containing $10^{7}$ logarithmically growing Sf9 cells. After $1 \mathrm{~h}$ the medium was removed and the cells were covered with $6 \mathrm{ml}$ of low melting agarose containing X-Gal. Starting 3 days later, the petri dishes were inspected for the appearance of blue plaques, generated by X-Gal metabolized by the protein expressed by the reconstituted lacZ gene. Blue plaques containing the recombinant virus were cut out and put into insect cell medium to elute the virus particles. This medium containing the virus was then used for subsequent infections of Sf9 cells.

Preparation of cell extracts. PBS-washed cells were lysed in RIPA buffer (50 mM Tris/ $\mathrm{HCl}, \mathrm{pH} 7.4$, $500 \mathrm{mM} \mathrm{NaCl}, 1 \%$ Nonidet-P40, 0.5\% Na-deoxycholate, $0.1 \%$ SDS, $0.05 \% \mathrm{NaN}_{3}, 1 \mathrm{mM}$ phenylmethylsulfonyl fluoride (PMSF)) for $20 \mathrm{~min}$ at $+4^{\circ} \mathrm{C}$. For affinity purification of baculovirally expressed proteins, insect cells were lysed directly in native binding buffer provided in the X-press purification Kit (Invitrogen Life Technologies, San Diego, CA, USA), and cell structures were destroyed by repeated freeze/ thaw procedure. The cell suspension was spun off. Clear supernatant was used for further analysis. The protein concentration of cell extracts was determined by the DC assay (Bio-Rad Laboratories, Richmond, CA, USA) with bovine serum albumin as standard.

Affinity chromatography on Ni-agarose. Cell lysates prepared in native binding buffer were loaded on preequilibrated Ni-agarose beads (ProBond, Invitrogen Life Technologies, San Diego, CA, USA) and purification of recombinant proteins was performed according to the manufacturer's protocol (Wesierska-Gadek et al., 2003b). Recombinant proteins were eluted from the Ni-beads with the native elution buffer at $\mathrm{pH} 4.0$ and immediately neutralized.

Pull down assay. To examine the effect of p53 phosphorylation on its binding ability to PARP-1, a pull down assay was performed (Wesierska-Gadek et al., 2003b). Full-length His-tagged PARP-1 was immobilized on $\mathrm{Ni}$-beads and then human native p53 lacking the His-tag-sequence was loaded. Before loading, p53 was incubated with calf intestine phosphatase or, alternatively, left untreated. After thorough washing steps, bound p53 was eluted stepwise and then analyzed by immunoblotting. Sequential eluates were designated eluate 1, eluate 2 etc.

Immunoprecipitation. Immunoprecipitation was performed as previously described in detail (Wesierska-Gadek et al., 1996a). Equal protein amounts of each cell lysate $(150 \mu \mathrm{g}$ protein) were used for incubation with the anti-p53 antibody CM1 and for incubation with anti-PARP-1 antibodies in a final volume of $100 \mu \mathrm{l}$. Immune complexes were then purified by affinity chromatography on prewashed Gamma-Bind Sepharose beads $(40 \mu \mathrm{l})$ (Pharmacia, Uppsala, Sweden) and eluted sequentially 3 times with SDS sample buffer. Sequential eluates were designated eluate 1, eluate 2 etc. Each eluate was then divided and loaded on two gels. One gel was used for immunoblotting with anti-p53 antibodies and the second for incubation with anti-PARP-1 antibodies.

Electrophoretic separation of proteins. Proteins were separated by 1D-PAGE on $10 \%$ or $15 \%$ SDS-gels as described by Laemmli (1970) or by 2D-PAGE according to O'Farrell (1975) with some modifications (Wesierska-Gadek et al., 2003b). In the lysis buffer and gel mixture NP-40 was replaced with 3-[(3-cholamidopropyl)dimethyl-ammonio]-1propane sulfonate (Bio-Rad, Richmond, CA, USA). To achieve good resolution of proteins within the $\mathrm{pH}$ range $4-8$ of isoelectrofocusing (IEF) gels, carrier ampholytes with broad (3.5-10) and narrow (4-8) $\mathrm{pH}$ ranges (BDH, Poole, England) were mixed at a ratio of 1:4. For calibration, BSA and two isoforms of carbonic anhydrase were used as markers. In the second dimension, samples were run on $10 \%$ SDS slab gels. After electrophoretic separation proteins 
were detected by silver staining of gels, or they were electrophoretically transferred onto a polyvinylidene difluoride membrane (PVDF) (Amersham International, Little Chalfont, Buckinghamshire, UK).

Immunoblotting. Proteins resolved by 1D- or 2D-PAGE and transferred onto a PVDF membrane were then stained with Ponceau $S$, saturated and incubated with specific primary antibodies diluted to a final concentration of 1:2000. The immune complexes were detected with appropriate peroxidaseconjugated secondary antibodies and the enhanced chemiluminescence detection reagent ECL+ (Amersham International, Little Chalfont, Buckinghamshire, UK).

\section{RESULTS}

Baculovirally expressed recombinant p53 protein is phosphorylated

We cloned human wt p53 and poly(ADP-ribose) polymerase-1 (PARP-1) into a baculovirus as full length proteins as well as their truncated fragments encompassing functional domains. Cellular p53 is a phosphoprotein and the phosphorylation regulates its main features: stability and activity. To prove the modification status of human wt p53 protein expressed in Sf9 insect cells we used two independent approaches. In the first approach, cell lysates separated by 2D-PAGE were analyzed by immunoblotting. As shown in Fig. 1, incubation with monoclonal anti-p53 antibodies visualized only a series of protein spots at approx. $55 \mathrm{kDa}$ indicating that the reactive antigen consists of several isoforms differing in their charge. However, these results did not provide information on the type and sites of the posttranslational modifications.

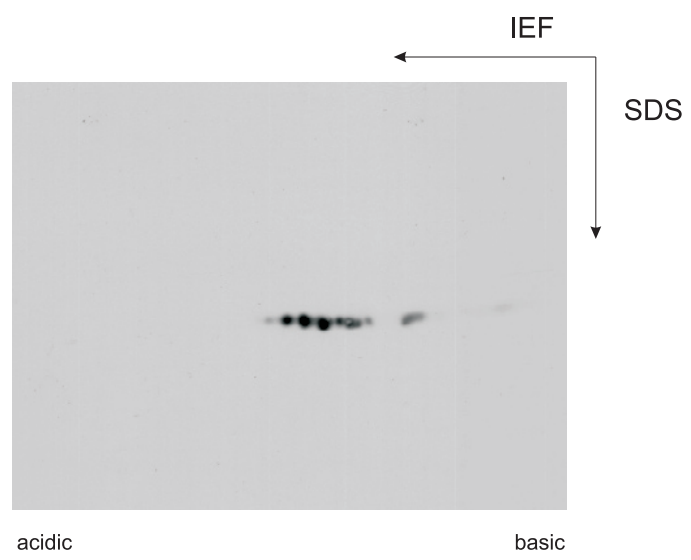

Figure 1. Baculovirally expressed human wt p53 consists of several isoforms.

Sf9 cell lysate was resolved by 2D-PAGE, then proteins were transferred onto PVDF and incubated with the antip53 antibody PAb421.
In the second approach, we examined sitespecific phosphorylation of the human recombinant protein using phospho-specific anti-p53 antibodies. To exclude the possibility that the His-tag sequence might affect the type and extent of p53 modification, we loaded two different p53 recombinants: tagged and untagged p53 protein and tested it with different phospho-specific antibodies. To prove equal protein loading the blots were then sequentially incubated with DO-1 antibodies recognizing p53 irrespective of its modification status.

Immunoblotting using phospho-specific antibodies revealed that p53 protein expressed in insect cells was phosphorylated at multiple sites (Fig. 2). Strong phosphorylation of serine in positions 15, 20, 46 and 392 was detected. Serine at position 37 was only weakly modified. The strongest modification was detected at Ser392 (Fig. 2). To achieve a satisfying resolution of the reactive band, we had to use an approximately five-fold lower concentration of the anti-P-Ser392 antibody (Fig. 2). The His-tag sequence at the $\mathrm{NH}_{2}$-terminus of p53 protein had no detectable effect on its phosphorylation status.

\section{Verification of the generated p53 recombinant pro- teins by specific antibodies}

The identity of the generated human p53 recombinant proteins was verified by immunoblotting using specific antibodies. Full length and distinct truncated p53 proteins were separated on duplicate blots and incubated with antibodies recognizing different epitopes localized within distinct functional domains. As shown in Fig. 3A the monoclonal antibody DO-1 recognized solely full length p53 protein as well as its $\mathrm{NH}_{2}$-terminal fragment aa 1-101 and failed to react with other fragments. PAb421 reacted with full length p53 and its $\mathrm{COOH}$-terminal parts aa 102-393 and aa 292-393, but did not react with the $\mathrm{NH}_{2}$-terminal fragment or the core domain. Finally, PAb240 recognized the truncated protein encompassing the core domain aa 102-292. The results of the immunoblotting experiments based on the size of the reactive p53 band and the reactivity with defined monoclonal antibodies confirmed the identity of the generated truncated p53 proteins.

\section{The $\mathrm{NH}_{2}$-terminal domain of $\mathrm{p} 53$ is dispensable for binding to PARP-1}

The complex formation between p53 and PARP-1 was described previously by us and other groups. However, no information was available about which domain(s) of p53 protein is involved in the binding to PARP-1. We addressed this issue and co-expressed full length PARP-1 with different truncated p53 proteins in insect cells. Then reciprocal immunoprecipitation experiments were performed 


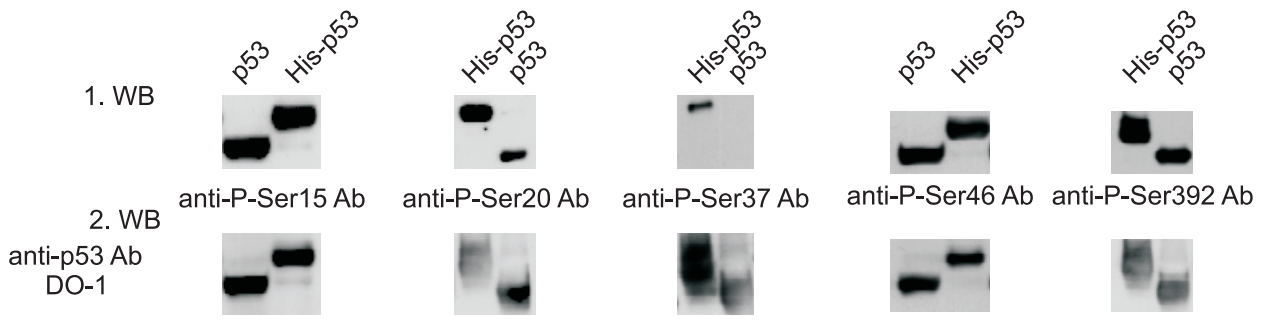

Figure 2. Detection of site-specific phosphorylation of human wt p53 protein expressed in insect cells.

Sf9 cells expressing His-tagged and untagged human wt p53 protein were lysed and analyzed by immunoblotting using phospho-specific anti-p53 antibodies and then they were incubated with monoclonal anti-p53 DO-1 antibodies to detect total p53 protein. WB, Western blot.

using anti-p53 and anti-PARP-1 antibodies. Interestingly, the $\mathrm{NH}_{2}$-terminal fragment of p53 failed to bind to PARP-1 (Fig. 3). No p53-specific signal could be detected after precipitation of the samples containing PARP-1 and the aa 1-102 p53 fragment with anti-PARP-1 antibodies (Fig. 3B). The monoclonal anti-p53 DO-1 antibody which reacts with an epitope within the $\mathrm{NH}_{2}$-terminal part of p53 protein recognized only full length p53 coprecipitated by anti-PARP-1 polyclonal antibody (Fig. 3B). Control experiments (input) revealed that, as expected,

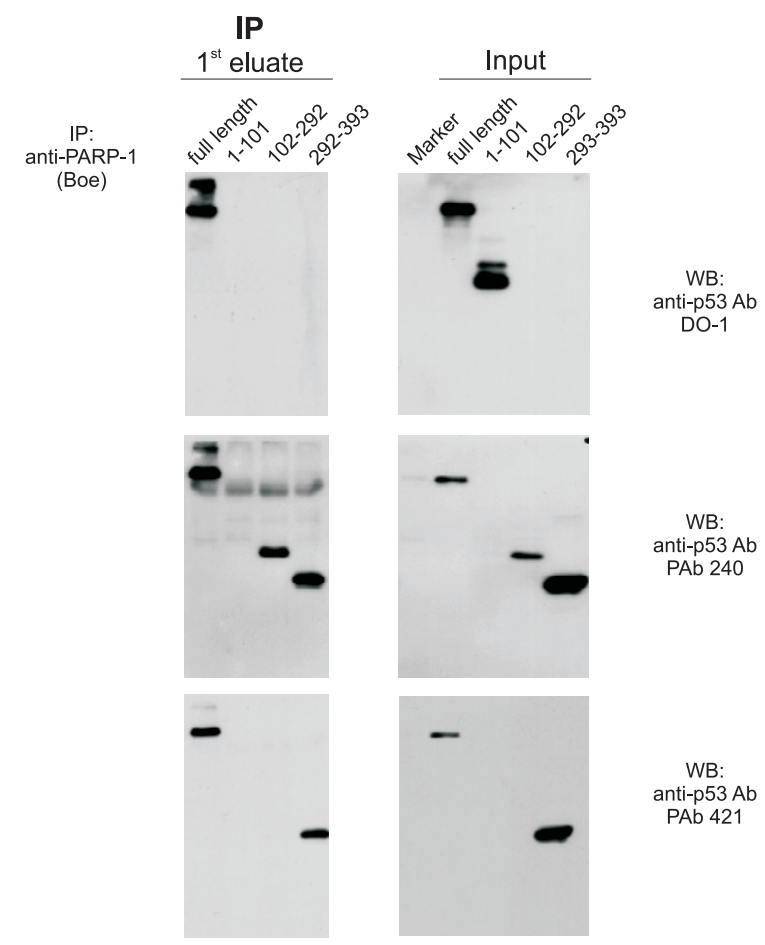

Figure 3. The amino-terminus of p53 is dispensable for binding with PARP-1.

Cell lysates obtained from Sf9 cells infected with a baculovirus encoding full length p53 and its distinc functional domains (input) were separated on 15\% SDS gels and analyzed by immunoblotting using different anti-p53 antibodies (right panel). Cell lysates obtained from Sf9 cells infected with baculovirus encoding full length PARP-1 in combination with full length p53 or its truncated proteins were precipitated (IP) using anti-PARP-1 antibodies. The immune complexes were analyzed by immunoblotting (WB) using distinct anti-p53 antibodies (left panel). the aa 1-102 p53 fragment was present in the cell lysate.

\section{Phosphorylation of p53 protein is essential for its binding to PARP-1}

In the next step we assessed the role of p53 phosphorylation for complex formation with PARP1. We immobilized His-tagged PARP-1 on Ni-columns and performed a pull down assay by loading untagged p53 protein. We compared the binding of control (untreated) and dephosphorylated p53 protein. Control p53 was bound to the immobilized PARP-1 and was eluted stepwise. Residual p53 molecules were released during the third and fourth elution (Fig. 4, eluate 3 and 4). However, after phosphatase treatment no p53 was bound to the column and for this reason no p53 was eluted. A p53 signal was detected solely in the flow-through fraction (not shown).

\section{DISCUSSION}

Due to its central role in the regulation of the cell cycle and in the decision to induce apoptosis or cell cycle arrest in damaged cells, the tumor sup-

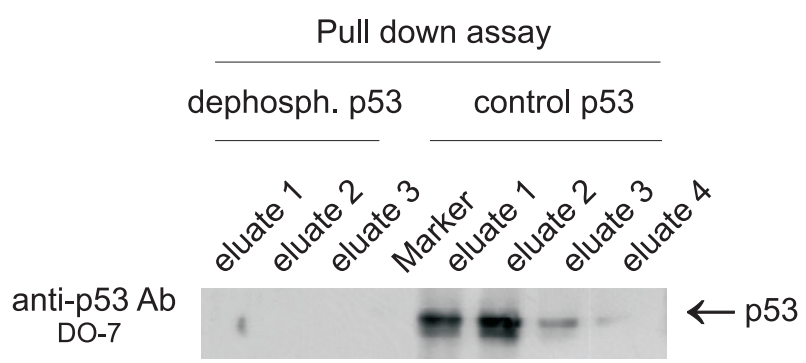

Figure 4. Dephosphorylation of p53 abolishes its binding to PARP-1.

Control native and dephosphorylated untagged p53 samples were loaded on Ni-beads on which His-tagged PARP-1 has been immobilized. The beads were washed thoroughly with wash buffer and then they were eluted stepwise at low $\mathrm{pH}$. Immediately after elution, samples were neutralized and analyzed by immunoblotting after separation on a $10 \%$ SDS gel. 


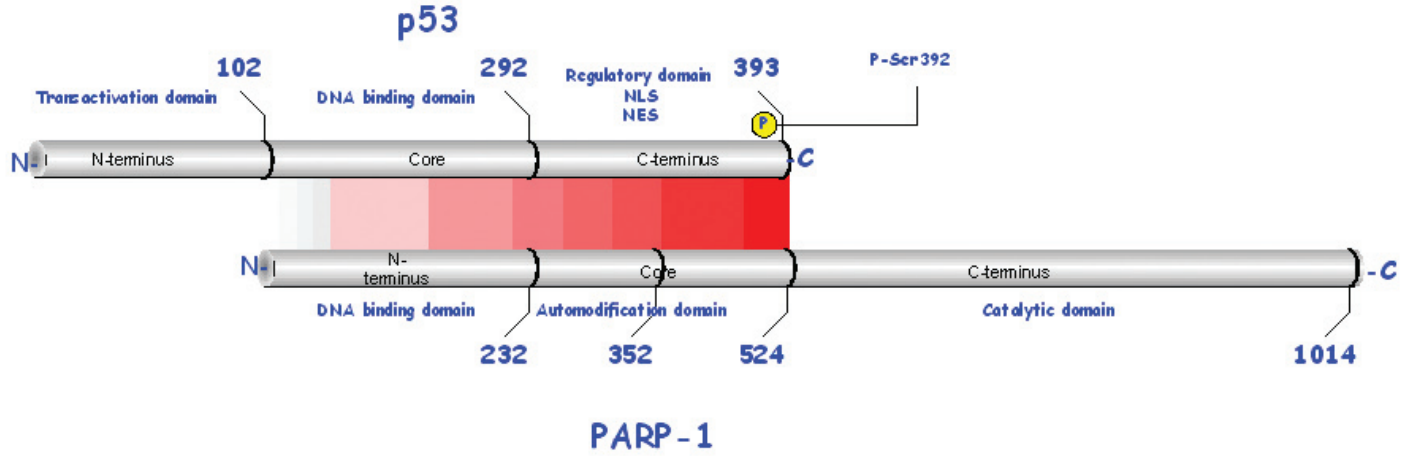

Figure 5. A model depicting the interaction between p53 and PARP-1.

Distinct protein domains of p53 protein and PARP-1 are schematically shown. NLS, nuclear localization signal; NES, nuclear export signal. Hatching indicates the domains involved in complex formation.

pressor protein p53 has been the target of many investigations during the last two decades.

Unfortunately, attempts to overexpress wt p53 in mammalian cells or in yeast have not been very successful because of its detrimental effect on cell growth and viability. Overexpressed wt p53 protein activates within a short time a number of target genes resulting in the induction of cell cycle arrest and massive apoptosis. Since PARP-1 is one of the key proteins regulating the stability and activity of p53 it was of high interest to explore the domains of both proteins responsible for the complex formation. However, an investigation of the interaction between wt p53 and PARP-1 in mammalian cells was difficult because during p53-induced apoptosis PARP-1 is one of the major targets of the activated apoptosome. Therefore, we decided to use the baculovirus expression system in insect cells to express the recombinant protein for two reasons. First, baculovirally expressed recombinant proteins exhibit a phosphorylation pattern resembling that in mammalian cells. Additionally, also other types of posttranslational modifications (e.g. glycosylation and myristoylation) that influence the properties of a protein are similar in insect and mammalian cells (O'Reilly et al., 1994). In contrast, a bacterial expression system cannot provide a properly modified protein. The central role of phosphorylation of p53 protein in its regulation has been illustrated in many studies. Secondly, according to the features of the baculovirus expressions system, the transcription of p53 downstream genes is not activated in insect cells and therefore the cells do not undergo apoptosis and grow exponentially until the virally induced lysis.

As we could show in earlier studies (Wesierska-Gadek et al., 2003a; 2003b), the central and the carboxy-terminal domain of p53 are necessary and sufficient to enable the protein to bind to PARP-1. In turn, the amino-terminal and central domains of PARP-1 are crucial for the ability of the protein to engage in heterodimerization with p53. In our experiments we employed specific phosphoserine antibodies against p53 phosphoprotein to explore the phosphorylation sites relevant to the dimerization properties of the protein. The inability of dephosphorylated p53 to bind PARP-1 made it evident that this posttranslational modification is a prerequisite to the complex formation of p53. The serine that turned out to be of fundamental importance for the dimerization of p53 is located at position 392 at the extreme carboxy-terminus of the protein.

Most importantly, the nuclear export signal of p53 is located in this region (Stommel et al., 1999) and the phosphorylation of the protein at this site, which confers the ability to dimerize with PARP-1, renders p53 resistant to nuclear export (WesierskaGadek et al., 2000a). Therefore, the protein cannot be degraded via the ubiquitin dependent pathway and its presence in the nucleus allows it to exert its function as a transcription factor. The results we gained in our studies clearly support this explanation. The biological significance of this regulation is also supported by the notion that in many cancer cells the activity of p53 is abrogated by exclusion of the protein from the nucleus. In cells in which PARP-1 is inactivated, the p53 level is decreased and the expression of p53-dependent genes is also affected (WesierskaGadek et al., 1999). In contrast, overexpression of PARP-1 leads to stabilization of the p53 protein and, at the cellular level, this leads to a stronger up-regulation of p53 targets (Wesierska-Gadek \& Schmid, 2000; 2001). As a consequence, cells which are in the $G_{1}$ phase of the cell cycle exhibit a pronounced delay in their re-entry into the cell cycle which leads to downregulated cell growth.

Our present study shows the basis of the regulation of the dimerization of p53 protein and PARP-1 and maps the domains and phosphorylation sites most important for this interaction. It is evident that PARP-1 plays a fundamental role in the stability, activity and localization of p53. Since it is clear that posttranslational modifications other than phosphorylation are also of central importance for p53 activity and stability it remains an important goal to elucidate the role of those modifications in upcoming investigations. 


\section{REFERENCES}

Apella E, Anderson CW (2001) Post-translational modifications and activation of p53 by genotoxic stresses. Eur J Biochem 268: 2764-2772.

Bargonetti J, Manfredi JJ (2002) Multiple roles of the tumor suppressor p53. Curr Opin Oncol 14: 86-91.

Berger NA (1985) Poly(ADP-ribose) in the cellular response to DNA damage. Radiat Res 101: 4-15.

Blagosklonny MV (2002) P53: An ubiquitous target of anticancer drugs. Int J Cancer 98: 161-166.

D'Amours D, Desnoyers S, D'Silvia I, Poirier GG (1999) Poly(ADP-ribosyl)ation reactions in the regulations of nuclear functions. Biochem I 342: 249-268.

de Murcia G, Menissier-de Murcia J (1994) Poly(ADP-ribose) polymerase: a molecular nick-sensor. Trends Biochem Sci 19: 172-176.

Donehower LA (2002) Does p53 affect organismal aging? J Cell Physiol 192: 23-33.

el-Deiry WS, Tokino T, Velculescu VE, Levy DB, Parsons R, Trent JM, Lin D, Mercer WE, Kinzler KW, Vogelstein B (1993) WAF1, a potential mediator of p53 tumor suppression. Cell 75: 817-825.

Fuchs SY, Adler V, Buschmann T, Yin Z, Wu X, Jones SN Ronai Z (1998) JNK targets p53 ubiquitination and degradation in nonstressed cells. Genes Dev 12: 2658-2663.

Haupt Y, Maya R, Kazaz A, Oren M (1997) Mdm2 promotes the rapid degradation of p53. Nature 387: 296299.

Kubbutat MH, Jones SN, Vousden KH (1997) Regulation of p53 stability by Mdm2. Nature 387: 299-303.

Laemmli UK (1970) Cleavage of structural proteins during the assembly of the head of the bacteriophage $\mathrm{T}_{4}$. $\mathrm{Na}$ ture 227: 680-685.

Maier B, Gluba W, Bernier B, Turner T, Mohammad K Guise T, Sutherland A, Thorner M, Scrable H (2004) Modulation of mammalian life span by a short isoform of p53. Genes Dev 18: 306-319.

Momand J, Zambetti G, Olson D, George D, Levine AJ (1992) The Mdm2 oncogene product forms a complex with the p53 protein and inhibits p53-mediated transactivation. Cell 69: 1237-1245.

Mowat MR (1998) p53 in tumor progression: life, death, and everything. Adv Cancer Res 74: 25-48.

O'Farell PH (1975) High resolution two-dimensional electrophoresis of proteins. J Biol Chem 250: 4007-4021.
O'Reilly DR, Miller LK, Luckow VA (1994) Baculovirus Expression Vectors: A Laboratory Manual. Freeman, New York.

Shaulsky G, Ben-Ze'ev A, Rotter V (1990) Subcellular distribution of the p53 protein during the cell cycle of Balb/c 3T3 cells. Oncogene 5: 1707-1711.

Stommel JM, Marchenko ND, Jimenez GS, Moll UM, Hope TJ, Wahl GM (1999) A leucine-rich nuclear export signal in the p53 tetramerization domain: regulation of subcellular localization and p53 activity by NES masking. $E M B O J$ 18: $1660-1672$.

Wesierska-Gadek J, Schmid G (2000) Overexpressed poly(ADP-ribose) polymerase delays the release of rat cells from p53-mediated $G_{1}$ checkpoint. J Cell Biochem 80: 85-103.

Wesierska-Gadek J, Schmid G (2001) Poly(ADP-ribose) polymerase-1 regulates the stability of the wild-type p53 protein. Cell Mol Biol Lett 6: 117-140.

Wesierska-Gadek J, Bugajska-Schretter A, Cerni C (1996a) ADP-ribosylation of p53 tumor suppressor protein: mutant but not wild-type p53 is modified. J Cell Biochem 62: 90-101.

Wesierska-Gadek J, Schmid G, Cerni C (1996b) ADP-ribosylation of wild-type p53 in vitro: binding of p53 protein to specific p53 consensus sequence prevents its modification. Biochem Biophys Res Commun 224: 96-102.

Wesierska-Gadek J, Wang ZQ, Schmid G (1999) Reduced stability of regularly spliced but not alternatively spliced p53 protein in PARP-deficient mouse fibroblasts. Cancer Res 59: 28-34.

Wesierska-Gadek J, Bohrn E, Herceg Z, Wang Z-Q, Wurzer G (2000) Differential susceptibility of normal and PARP knock-out mouse fibroblasts to proteasome inhibitors. J Cell Biochem 78: 681-696.

Wesierska-Gadek J, Wojciechowski J, Schmid G (2003a) Central and carboxy-terminal regions of human p53 protein are essential for interaction and complex formation with PARP-1. J Cell Biochem 89: 220-232.

Wesierska-Gadek J, Wojciechowski J, Schmid G (2003b) Phosphorylation regulates the interaction and complex formation between wt p53 protein and PARP-1. J Cell Biochem 89: 1260-1284. 